\title{
La influencia de la correcta consideración climática en los certificados energéticos realizados en Canarias
}

\section{The influence of the correct climatic consideration on the energy certificates in the Canary Islands}

\author{
$\underline{\text { E. Martín del Toro }}^{(*)}$
}

RESUMEN

El certificado energético se presenta como una herramienta de gran utilidad al ofrecer información sobre la eficiencia energética, los consumos energéticos asociados y las emisiones de $\mathrm{CO}_{2}$ que dichos consumos generan. Pero su aplicación en Canarias se ha encontrado con problemas, ya que las particularidades propias del archipiélago dan lugar, por un lado a que el clima de las islas sea muy diferente del resto de España y por otro lado el encontrarse marcado por microclimas que originan una importante variedad climática en una pequeña extensión de superficie. Este aspecto, que no se vio recogido en un primer momento por el CTE, ha ido posteriormente ajustándose. Este trabajo analiza las discrepancias producidas entres las distintas zonas climáticas propuestas en las diferentes áreas del archipiélago para conocer cuáles se acercan más a las condiciones reales de las islas y las implicaciones que estas divergencias han provocado, como situaciones con resultados incoherentes.

Palabras clave: Certificado de eficiencia energética, zona climática, Canarias, CLIMCAN.

\section{ABSTRACT}

The energy certificate is a very useful tool, as it provides information on energy efficiency, the associated energy consumption and the $\mathrm{CO} 2$ emissions generated by these consumptions. But its application in the Canary Islands has encountered several problems. The particular conditions of the archipelago give rise to the fact that the climate of the islands is very different from the rest of Spain and also generate microclimates that develop an important climatic variety in a small area. This aspect, which was not initially taken into consideration by the ETC, has been progressively adjusted. This paper analyses the discrepancies between the different climatic zones proposed in the different areas of the archipelago to find out which are closer to the real conditions of the islands and the implications that these divergences have caused, such as situations with incoherent results.

Keywords: Energy efficiency certificate, climatic zone, Canary Island, CLIMCAN.

(*) Doctor arquitecto. Grupo de investigación ARQUITECTURA Y PAISAJE de la Universidad de Las Palmas de Gran Canaria. Las Palmas de Gran Canaria (España).

Persona de contacto/Corresponding author: eduardo.martindeltoro@ulpgc.es (E. Martín del Toro).

ORCID: https://orcid.org/oooo-0001-9609-2947 (E. Martín del Toro).

Cómo citar este artículo/Citation: Martín del Toro, E. (2019). La influencia de la correcta consideración climática en los certificados energéticos realizados en Canarias. Informes de la Construcción, 71(556): e310. https://doi.org/10.3989/ic.66351.

Copyright: (c) 2019 CSIC. Este es un artículo de acceso abierto distribuido bajo los términos de la licencia de uso y distribución Creative Commons Reconocimiento 4.o Internacional (CC BY 4.0). 


\section{INTRODUCCIÓN}

La desmedida simplificación climática realizada por el HE 2006 (1), asignando sólo dos zonas climáticas a un archipiélago marcado por sus tremendas variaciones térmicas, que pueden llevar a situaciones tan extremas como que suceda de forma simultánea se den niebla y temperaturas frescas en La Laguna y, a unos pocos kilómetros, en la Punta del Hidalgo se encuentre un sol radiante con altas temperaturas, provoca, por ejemplo, que los programas de certificación energética asignen demanda de calefacción en el conjunto del archipiélago, y la consiguiente instalación por defecto de un equipo de calefacción para satisfacer dicha demanda, lo que resulta absurdo en la práctica totalidad del litoral canario. Como respuesta a este problema se produjeron diversas reacciones:

En primer lugar, el programa reconocido para calificación de viviendas y pequeños y medianos terciarios, CALENER versión VYP, anuló esta demanda de calefacción cuando en lugar de introducir el clima genérico A3, se indicaba que el edificio de estudio se encontraba en una de los dos capitales de provincia: Las Palmas de Gran Canaria o Santa Cruz de Tenerife. El Gobierno de Canarias, por medio de una circular (2), “admitirá de forma transitoria, hasta la publicación y reconocimiento de una zonificación más ajustada a la realidad del archipiélago canario, la utilización de las capitales de provincia como "Localidad" para todas las localidades cuya cota de referencia sea inferior a los 200 metros".

Esta corrección no tuvo reflejo en las herramientas simplificadas como $\mathrm{CE}^{3} \mathrm{X}$ o $\mathrm{CE}_{3}$, que mantuvieron la demanda de calefacción en el resultado de los certificados energético, aún cuando se indicaba como situación del inmueble cualquiera de las dos capitales de provincia.

Posteriormente, y a instancias del Gobierno de Canarias, se solicita para la tramitación como Documento Reconocido del Código Técnico de la Edificación de la Guía CLIMCAN-O10 "Caracterización climática de las islas canarias para la aplicación del Código Técnico de la Edificación" y de su aplicación informática (3), que es aprobado en octubre de 2011 y que detalla una nueva distribución espacial de cada una de las zonas climáticas en función de los umbrales del CTE, ampliando de 2 a 7 zonas climáticas en el archipiélago: A3, A4, B3, B4, C2, C3 y D1.

La entrada en vigor del CLIMCAN-010 fue controvertida, con un fuerte rechazo por parte del Colegio Oficial de Arquitectos de Canarias (COAC) ya que en esos momentos, desde esa institución, se impulsaba una propuesta diferente de metodología (4), con otra estrategia de adaptación de las características del clima de Canarias, para una zonificación alternativa, tanto a la expresada en el HE 2006 como en ese documento, para conseguir alcanzar el confort térmico de los edificios minimizando las demandas energéticas que mitiguen la necesidad del empleo de sistemas activos de climatización, en base a la incuestionable benignidad del clima canario, cuyas características son únicas en todo el territorio español y por consiguiente requieren un trato claramente diferenciado.

La principal causa en la reclamación del COAC contra el CLIMCAN-010 se fundamenta en que a la hora de realizar la nueva zonificación climática "no se contempla la humedad, que es uno de los factores clave en el confort (junto con la temperatura) y que en Canarias es especialmente relevante, con medias de en torno al 70\% y máximas de hasta el 90\%” (5) ya que sólo se tienen en cuenta la temperatura, la radiación solar y el número de horas de sol. Otros aspectos son también la laxitud en la exigencia de protecciones solares o la eficacia no valorada de la arquitectura bioclimática.

En contraposición, el COAC propuso en el año 2006 una propuesta de Documento Reconocido -que nunca fue aprobado- basada en tres aspectos generales:

1. Un mayor rigor en la determinación de las zonas climáticas, que contemple particularidades del clima canario como es la humedad y las singulares características de las zonas afectadas por el mar de nubes en las vertientes norte de las islas.

2. Favorecer (y valorar para la Certificación Energética de los Edificios) la utilización de las estrategias de la Arquitectura Solar Pasiva (o bioclimática) como principal sistema para conseguir la eficiencia energética en la edificación, aprovechando lo benigno del clima del archipiélago y su suave oscilación térmica estacional.

3. Una mayor exigencia en las protecciones solares, ya que el mayor problema en Canarias es de sobrecalentamientos, no de pérdidas de calor en régimen de calefacción.

Finalmente, la entrada en vigor de la actualización del DBHE 2013 (6) modifica las consideraciones globales de las zonas climáticas e introduce una nueva zona climática específica para canarias, la alfa3 para las localidades situadas por debajo de los $350 \mathrm{~m}$ de altitud, que se acerca más a las condiciones costeras del archipiélago, aunque sigue sin existir consideraciones entre vertientes norte y sur.

Por tanto, todas estas inexactitudes y cambios sucesivos de las zonas climáticas en el archipiélago han provocado que el resultado de los certificados energéticos sea incierto y muy diferente en relación a cual sea la hipótesis de zona climática tomada, aumentando la desconfianza de técnicos y sociedad en general.

\section{METODOLOGÍA}

La certificación energética de edificios surge como una herramienta para poner a disposición de los compradores o usuarios de los edificios un documento que informe de forma objetiva sobre su eficiencia energética con el fin de que los propietarios o arrendatarios del edificio puedan comparar y evaluar su eficiencia energética.

A pesar de que dicha herramienta se encuentra en vigor en España desde el 30 de abril de 2007 para los edificios nuevos, gracias al Real Decreto 47/2007, y posteriormente ha ampliado su rango también a los edificios existentes, su implantación en edificios nuevos es de escasa entidad en comparación con los existentes, suponiendo estos últimos el 99\% de los certificados energéticos registrados en la Comunidad Autónoma de Canarias a 31 de diciembre de 2017 (7).

Este hecho se explica por varias razones: Por un lado, el Real Decreto 47/2007 no tuvo gran efecto tras su entrada en vigor debido, por un lado a la falta de conocimiento por parte de los técnicos dada la poca difusión que hubo en torno a su implantación y al retraso de las Comunidades Autónomas en el cumplimiento de sus obligaciones como entidades de registro y control de los certificados, dándose muchos casos en los que a pesar de estar la normativa en vigor, la Comunidad Autónoma aún no contaba con el prescriptivo registro. Y por otro lado, por el efecto que la crisis de la construcción, que ha generado una 
reducción importante en el número de proyectos nuevos visados, en general en todo el territorio español y en particular en el archipiélago canario - algo que comienza a revertirse- en relación a la gran cantidad de edificios existentes que componen la planta edificada de nuestro país, aunque sólo tengamos en cuenta los que están en disposición de venta o alquiler, únicos obligados por el Real Decreto 235/2013 para su certificación.

Por otro lado, existen gran variedad de procedimientos para la certificación de edificios (8). Por una parte está el procedimiento general para la certificación energética de edificios en proyecto, terminados y existentes por medio del programa informático de referencia Herramienta unificada LIDER-CALENER (HULC), de iniciativa pública, para la calificación de eficiencia energética de todo tipo de edificios. Por otra parte, los procedimientos simplificados para la certificación energética de edificios existentes como los programas informáticos simplificados de iniciativa pública, para la calificación de eficiencia energética de edificios existentes - $\mathrm{CE}_{3}$ y $\mathrm{CE}^{3} \mathrm{X}$ - o el programa informático simplificado CERMA "Método Abreviado (CERMA)", de iniciativa privada, para la calificación de eficiencia energética de viviendas tanto nuevas como existentes.

Pero si atendemos al tipo de programa o herramienta informática empleado predominante por los técnicos certificadores para el cálculo del certificado energético de los edificios tenemos que existe una clara ventaja por el software desarrollado por Natural Climate Systems SA (UTE Miyabi y Fundación CENER) denominado $\mathrm{CE}^{3} \mathrm{X}$, siendo del orden del 96,59\% de todos los certificados registrados en Canarias, según datos facilitados por la Dirección General de Industria y Energía de Canarias.

En cuanto al tipo de edificio, nos centraremos en los de uso residencial vivienda dado que el número de certificados de esta tipología es del $89 \%$ frente al 11\% de uso terciario (7).

Por todo esto, y dado que la finalidad del trabajo de investigación era evaluar el impacto real que han supuesto los cambios producidos en las consideraciones climáticas en el resultado de los certificados energéticos realizados en la Comunidad Autónoma de Canarias, entendemos que los valores más significativos para este estudio, dado los datos analizados son los certificados de edificios existentes de tipología vivienda, realizados con el programa informático $\mathrm{CE}^{3} \mathrm{X}$, que recoge más del $80 \%$ de los certificados energéticos totales registrados en dicho archipiélago.
A pesar de que para el desarrollo de este estudio nos centremos en una herramienta simplificada de uso exclusivo para edificios existentes, sus resultados pueden ser extrapolables a los edificios nuevos, dado que la base metodológica de cálculo es la misma. Tanto es así, que existen herramientas simplificadas que permiten el certificado tanto de edificios nuevos como existentes, como CERMA. Por otro lado, la herramienta de estudio -el $\mathrm{CE}^{3} \mathrm{X}$ - se encuentra pendiente de aceptación como Documento Reconocido para la Certificación Energética de Edificios Nuevos por medio de la aplicación de un complemento, por lo que se espera que en poco tiempo esta herramienta se pueda emplear para la certificación de cualquier tipo de inmueble, ya sea su uso -vivienda, pequeño o gran terciario- y si son nuevos o existentes.

Una vez acotado el ámbito de estudio a los certificados energéticos de edificios existentes, con uso residencial vivienda y realizados mediante la herramienta informática $\mathrm{CE}^{3} \mathrm{X}$ (v1,3 y v2,3), nos queda definir los modelos tipos sobre los que realizar las simulaciones. Para ello, se tomarán como base los archivos de ejemplo que vienen con el propio programa, al tratarse de prototipos contrastados y así minimizar la posibilidad de desviaciones en los resultados por la posible entrada incorrecta de datos, pero adaptándolos a la realidad particular del archipiélago y sus particularidades tanto constructivas como de medios energéticos, en especial al modo de construir con materiales como el bloque de hormigón vibroprensado (definidos en la herramienta $\mathrm{CE}^{3} \mathrm{X}$ como "fábrica de bloque de picón") y las instalaciones por medio de caldera convencional individual con GLP (en Canarias no existe red de gas ciudad por lo que los equipos a gas emplean butano o propano, ambos Gas Licuado del Petróleo) o el aún más empleado termo eléctrico: "En Canarias, lo normal es contar únicamente con sistema individual para generación de agua caliente, siendo normalmente un termo eléctrico (efecto Joule). Sistema muy penalizado por los programas de cálculo debido a su bajo rendimiento" (9).

Dado que el programa cuenta con ejemplos de bloque de viviendas y vivienda dentro de bloque y tras comprobar que los resultados obtenidos en cada caso son muy similares, así como en el caso de realizar variantes sobre los mismos -como cambios de orientaciones o alturas-, se desarrolla a continuación el caso de bloque de viviendas.

De forma detallada los cambios producidos para realizar dicha adaptación son los que se exponen en la Tabla 1, a continuación:

Tabla 1. Modificaciones realizadas sobre el archivo de ejemplo "Bloque de viviendas" para adaptarlo a la realidad particular del archipiélago.

\begin{tabular}{|c|c|c|}
\hline Sección & Apartado & Modificación \\
\hline Datos administrativos & Localización e identificación del edificio & Se actualiza la localización a la zona de estudio \\
\hline Datos generales & Datos generales & Se actualiza la localización y zona climática a la zona de estudio* \\
\hline \multirow{4}{*}{ Envolvente térmica } & Muros & $\begin{array}{l}\text { Propiedades térmicas/Una hoja/Fábrica de bloques de picón } \\
\left(\mathrm{U} 1,69 \mathrm{~W} / \mathrm{m}^{2} \cdot \mathrm{K}\right)\end{array}$ \\
\hline & Cubierta & $\begin{array}{l}\text { Propiedades térmicas/Estimada/Cubierta plana/Unidireccional } \\
\left(\mathrm{U} 2,27 \mathrm{~W} / \mathrm{m}^{2} \cdot \mathrm{K}\right)\end{array}$ \\
\hline & Hueco/Lucernario & $\begin{array}{l}\text { Propiedades térmicas/Tipo de vidrio/simple } \\
\left(\mathrm{U} 5,7 \mathrm{O} \mathrm{W} / \mathrm{m}^{2} \cdot \mathrm{K}, \mathrm{g} 0,82 / \mathrm{o}, 75\right)^{* * *}\end{array}$ \\
\hline & Puente térmico & Se adaptan lo puentes térmicos a los cambios anteriormente indicados \\
\hline \multirow[b]{2}{*}{ Instalaciones } & Calefacción y ACS (40\%)/ Gas & GLP (P 24,o kW, $\varphi$ 53,60/56,8)** \\
\hline & Se añade Equipo de Sólo refrigeración & $\begin{array}{l}\text { Máquina frigorífica - Caudal Ref. Variable/Electricidad/Datos de rendi- } \\
\text { miento por defecto }(\varphi 90,00 / 100)^{* *}\end{array}$ \\
\hline
\end{tabular}

* Según Tabla 2.

** En caso de dos valores se corresponden el primero a la v1,3 y el segundo a la v2,3. 
A pesar de que los equipos de climatización no son comunes en los inmuebles residenciales canarias, ya sea porque la calefacción no es necesaria en las zonas costeras -por otro lado las más pobladas- y poco utilizadas en casi todo el resto de la isla a pesar de sí ser necesarias para un correcto confort (10) y que con el aire acondicionado pasa exactamente lo contrario, es decir, a pesar de que si existe demanda de refrigeración en zonas bajas del archipiélago, en algunos casos durante casi todo el año, culturalmente se considera un lujo propio de usos administrativos o turísticos, pero innecesario en viviendas. Dado que si existe demanda de climatización el programa asigna un equipo por defecto, se ha incluido equipos de calefacción y refrigeraciones todos los casos para así unificar condiciones y evitar dispersiones en los resultados, sobre todo si tenemos en cuenta que la versión $\mathrm{CE}^{3} \mathrm{X}$ versión 2 modifica los equipos por defecto con respecto a la versión 1: "Las características técnicas de los sistemas de sustitución se han modificado para adecuarlas a las exigencias reglamentarias establecidas en el RITE” (11).

Para poder abarcar un abanico suficientemente amplio y, por tanto, significativo tomamos como lugares de referencia donde situar los modelos de estudio, aquellos que se correspondan con cada una de las siete zonas climáticas que contempla el CLIMCAN, como muestra la Figura 1, siempre atendiendo a que en la correspondencia de las zonas climáticas asignadas según las dos versiones de documento básico de ahorro energético -2006 y 2013- también se incluyan todos las zonas climáticas asignadas para Canarias: dos en 2006 y cuatro en 2013.

Con todo esto tenemos que las simulaciones a realizar serán las resultantes de la combinación de las localizaciones expresadas en la Figura 1, con las tres zonificaciones climáticas asignadas para Canarias, tal como de expresa en la Tabla 2, a continuación.

\section{RESULTADOS}

Tras definir los modelos de estudios se procede a calcular la calificación energética de los mismos por medio de la herramienta informática $\mathrm{CE}_{3} \mathrm{X}$. Se han excluido los resultados de consumo energéticos del edificio debido a dos razones:

Por un lado, las primeras etiquetas energéticas sólo mostraban la calificación en relación a las emisiones de $\mathrm{CO}_{2}$, siendo el valor que aún hoy en día se tiene más en consideración por los ciudadanos.

Por otro lado, a partir del 14 de enero de 2016 se modifica el indicador de consumo de energía, adoptado como dicho indicador de consumo en la certificación, el de consumo de energía primaria no renovable (12).

Tabla 2. Relación de las condiciones de los modelos a simular mediante la herramienta informática CE3X.

\begin{tabular}{|c|c|c|c|c|c|c|c|c|}
\hline \multirow{3}{*}{ Archivo } & \multirow{3}{*}{ Situación } & \multirow{3}{*}{ Altitud (m) } & \multicolumn{6}{|c|}{ Zona climática } \\
\hline & & & \multicolumn{2}{|c|}{ HE 2006} & \multicolumn{2}{|c|}{ CLIMCAN } & \multicolumn{2}{|c|}{ HE 2013} \\
\hline & & & HE-1 & HE-4 & HE-1 & HE-4 & HE-1 & HE-4 \\
\hline Aro_edf_(6/C/13) & Arona & 630 & A3 & $\mathrm{V}$ & A3 & IV & A2 & $\mathrm{V}$ \\
\hline Res_edf_(6/C/13) & La Restinga & 15 & $\mathrm{~A} 3$ & $\mathrm{~V}$ & A4 & $\mathrm{V}$ & $\alpha 3$ & $\mathrm{~V}$ \\
\hline Esp_edf_(6/C/13) & La Esperanza & 879 & B3 & $\mathrm{V}$ & $\mathrm{B}_{3}$ & II & B2 & $\mathrm{V}$ \\
\hline Vil_edf_(6/C/13) & Vilaflor & 1400 & B3 & $\mathrm{V}$ & B4 & IV & $\mathrm{C} 2$ & $\mathrm{~V}$ \\
\hline Mal_edf_(6/C/13) & Malpaso & 1501 & B3 & $\mathrm{V}$ & $\mathrm{C} 2$ & IV & $\mathrm{C} 2$ & $\mathrm{~V}$ \\
\hline Sjl_edf_(6/C/13) & San José de Los Llanos & 1100 & B3 & $\mathrm{V}$ & $\mathrm{C}_{3}$ & II & $\mathrm{C} 2$ & $\mathrm{~V}$ \\
\hline Pct_edf_(6/C/13) & Parador de Cañadas del Teide & 2152 & B3 & $\mathrm{V}$ & D1 & $\mathrm{V}$ & $\mathrm{C}_{2}$ & $\mathrm{~V}$ \\
\hline
\end{tabular}

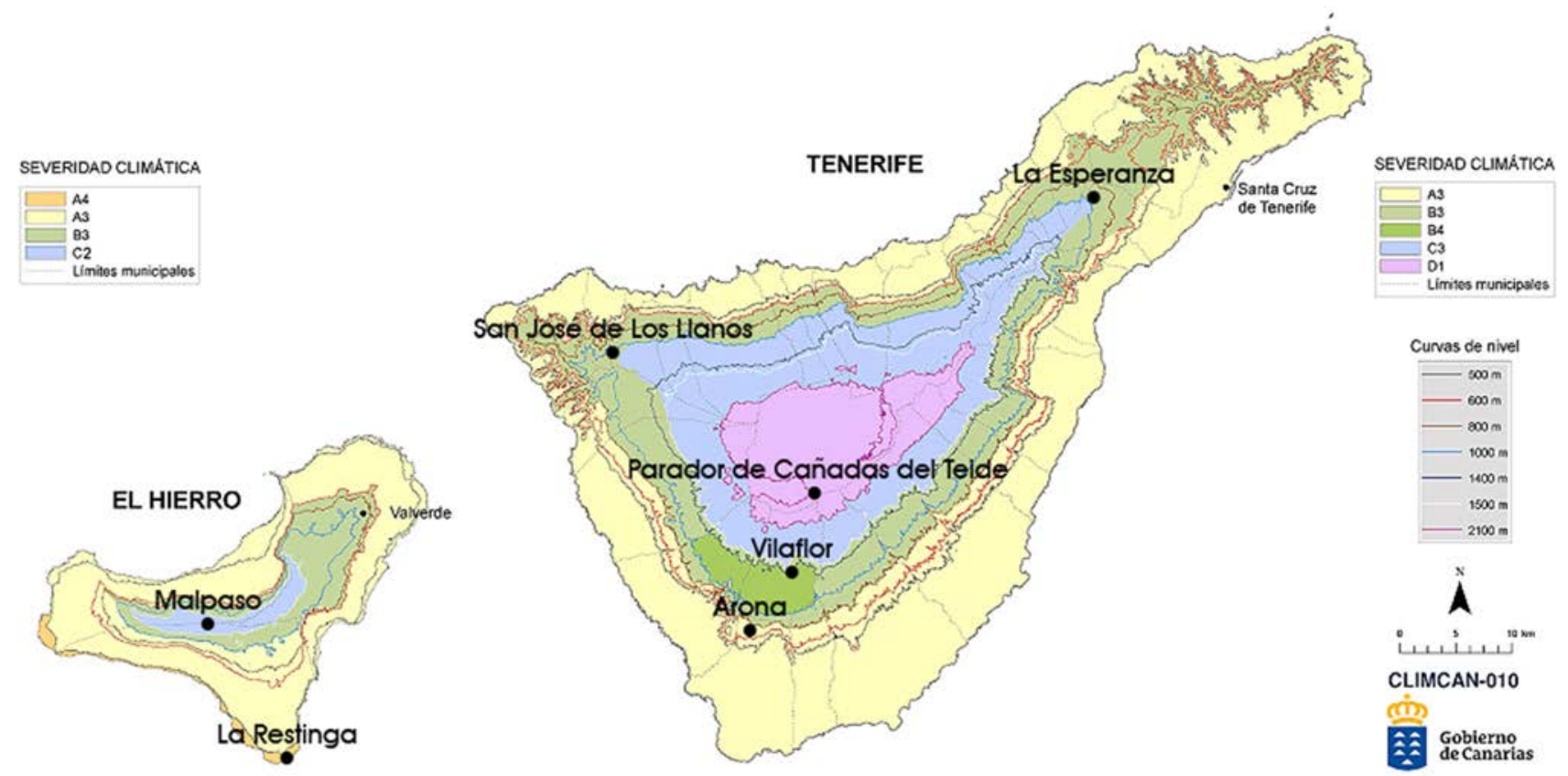

Figura 1. Situación geográfica hipotética de los modelos a calcular. Elaboración propia sobre los planos de severidad climática del CLIMCAN-010. 
También tenemos que tener en cuenta que hay ciertos factores que afectan, aunque en menor medida, a los resultados de emisiones de $\mathrm{CO}_{2}$, como son la actualización de los factores de emisión de $\mathrm{CO}_{2}$ y coeficientes de paso a energía primaria de diferentes fuentes de energía final, la modificación las características técnicas de los sistemas de sustitución o la actualización en el procedimiento para la obtención de las prestaciones medias estacionales de equipos y sistemas de producción de frío y calor.

Con todo esto, y de cara a trabajar con una correcta muestra que evite desviaciones de los resultados indeseadas, los valores más representativos para realizar la comparación de resultados generados a partir de la diferente zona climática considerada serán las demandas producidas, tanto en calefacción como en refrigeración.
Tras tener en cuenta las consideraciones anteriormente expuestas, se presentan los resultados en la Tabla 3 siguiente:

\section{ANÁLISIS DE LOS RESULTADOS}

Una vez obtenidos los resultados se procede al análisis comparativo que nos permitan obtener conclusiones relevantes. Para ello realizaremos la comparación de cada una de los modelos en cada uno de los enclaves propuestos, en base a la zona climática definida por cada una de las normativas estudiadas: HE 2016, CIMCAN y HE 2013.

Se puede observar en la Figura 2 que mientras los valores de los certificados realizados con las condiciones del HE 2006 y el CLIMCAN son idénticas, a pesar de que difieren en la Zona de radiación solar (zona V en el HE 2006 y zona IV en

Tabla 3. Compendio de resultados obtenidos tras el cálculo de la certificación energética de los modelos.

\begin{tabular}{|c|c|c|c|c|c|c|c|c|c|c|c|c|}
\hline \multirow{3}{*}{ Archivo } & \multicolumn{4}{|c|}{ Demanda } & \multicolumn{8}{|c|}{ Emisiones de dióxido de carbono } \\
\hline & \multicolumn{2}{|c|}{ Calefacción } & \multicolumn{2}{|c|}{ Refrigeración } & \multicolumn{2}{|c|}{ Globales } & \multicolumn{2}{|c|}{ Calefacción } & \multicolumn{2}{|c|}{ Refrigeración } & \multicolumn{2}{|c|}{ ACS } \\
\hline & $\mathbf{k W h} / \mathbf{m}^{2}$ año & Letra & $\mathbf{k W h} / \mathbf{m}^{2}$ año & Letra & $\mathbf{k g C O}_{2}$ & Letra & $\mathbf{k g C O}_{2}$ & Letra & $\mathbf{k g C O}_{2}$ & Letra & $\mathrm{kgCO}_{2}$ & Letra \\
\hline Aro_edf_6 & 20,89 & $\mathrm{E}$ & 28,77 & $\mathrm{~F}$ & 65,52 & $\mathrm{G}$ & 17,47 & $\mathrm{G}$ & $29,50^{2}$ & $\mathrm{G}$ & 18,55 & G \\
\hline Aro_edf_C & 20,89 & $\mathrm{E}$ & 28,77 & $\mathrm{~F}$ & 65,52 & G & 17,47 & $\mathrm{G}$ & 29,50 & G & 18,55 & G \\
\hline Aro_edf_13 & 33,0 & $\mathrm{E}$ & 2,9 & $\mathrm{~B}$ & 37,7 & $\mathrm{~F}$ & 21,25 & $\mathrm{E}$ & 21,25 & $\mathrm{C}$ & 14,30 & G \\
\hline Res_edf_6 & 20,89 & $\mathrm{E}$ & 28,77 & $\mathrm{~F}$ & 65,52 & G & 17,47 & G & 29,50 & G & 18,55 & G \\
\hline Res_edf_C & 24,43 & $\mathrm{E}$ & 33,84 & $\mathrm{~F}$ & 73,56 & G & 20,43 & G & 34,69 & G & 18,44 & $\mathrm{G}$ \\
\hline Res_edf_13 & $*$ & * & 15,0 & $\mathrm{D}$ & 25,0 & G & 0,00 & * & 10,98 & $\mathrm{~F}$ & 14,03 & G \\
\hline Esp_edf_6 & 47,76 & $\mathrm{E}$ & 20,68 & $\mathrm{E}$ & 79,90 & G & 39,93 & G & 21,20 & G & 18,76 & G \\
\hline Esp_edf_C & 47,76 & $\mathrm{E}$ & 20,68 & $\mathrm{E}$ & 79,90 & G & 39,93 & G & 21,20 & G & 18,76 & $\mathrm{G}$ \\
\hline Esp_edf_13 & 51,0 & $\mathrm{E}$ & 3,0 & B & 49,4 & $\mathrm{~F}$ & 32,84 & $\mathrm{E}$ & 2,14 & $\mathrm{C}$ & 14,41 & $\mathrm{G}$ \\
\hline Vil_edf_6 & 47,76 & $\mathrm{E}$ & 20,68 & $\mathrm{E}$ & 79,90 & G & 39,93 & G & 21,20 & G & 18,76 & $\mathrm{G}$ \\
\hline Vil_edf_C & 37,35 & $\mathrm{E}$ & 38,55 & $\mathrm{E}$ & 89,30 & G & 31,23 & G & 39,52 & G & 18,55 & $\mathrm{G}$ \\
\hline Vil_edf_13 & 90,8 & $\mathrm{E}$ & 2,9 & B & 75,3 & $\mathrm{~F}$ & 58,54 & $\mathrm{~F}$ & 2,10 & $\mathrm{C}$ & 14,67 & $\mathrm{G}$ \\
\hline Mal_edf_6 & 47,76 & $\mathrm{E}$ & 20,68 & $\mathrm{E}$ & 79,90 & G & 39,93 & G & 21,20 & G & 18,76 & G \\
\hline Mal_edf_C & 68,44 & $\mathrm{E}$ & 11,27 & $\mathrm{D}$ & 87,73 & G & 57,22 & G & 11,55 & G & 18,96 & $\mathrm{G}$ \\
\hline Mal_edf_13 & 90,8 & $\mathrm{E}$ & 2,9 & B & 75,3 & $\mathrm{~F}$ & 58,54 & $\mathrm{~F}$ & 2,10 & $\mathrm{C}$ & 14,67 & $\mathrm{G}$ \\
\hline Sjl_edf_6 & 47,76 & $\mathrm{E}$ & 20,68 & $\mathrm{E}$ & 79,90 & G & 39,93 & G & 21,20 & G & 18,76 & G \\
\hline Sjl_edf_C & 85,20 & $\mathrm{E}$ & 23,20 & $\mathrm{~F}$ & 114,09 & G & 71,24 & G & 23,78 & $\mathrm{G}$ & 19,07 & G \\
\hline Sjl_edf_13 & 90,8 & $\mathrm{E}$ & 2,9 & B & 75,3 & $\mathrm{~F}$ & 58,54 & $\mathrm{~F}$ & 2,10 & $\mathrm{C}$ & 14,67 & G \\
\hline Pct_edf_6 & 47,76 & $\mathrm{E}$ & 20,68 & $\mathrm{E}$ & 79,90 & G & 39,93 & $\mathrm{G}$ & 21,20 & $\mathrm{G}$ & 18,76 & G \\
\hline Pct_edf_C & 140,16 & $\mathrm{E}$ & 2,94 & * & 139,81 & $\mathrm{G}$ & 117,18 & $\mathrm{G}$ & 3,02 & * & 19,61 & $\mathrm{G}$ \\
\hline Pct_edf_13 & 90,8 & $\mathrm{E}$ & 2,9 & B & 75,3 & $\mathrm{~F}$ & 58,54 & $\mathrm{~F}$ & 2,10 & $\mathrm{C}$ & 14,67 & G \\
\hline
\end{tabular}

* No calificable.

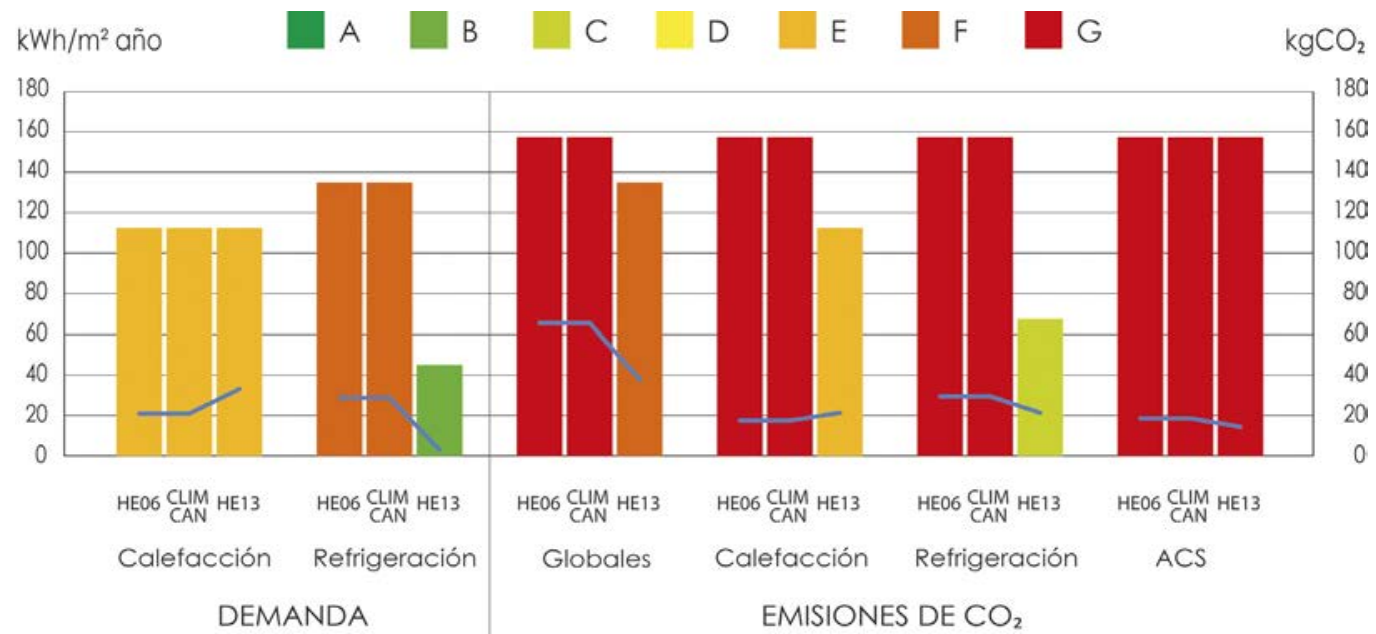

Figura 2. Comparativa de demandas y emisiones para un edificio situado en Arona. 
el CLIMCAN) cuando aplicamos los criterios del HE 2013 se produce un leve aumento de la demanda de calefacción y una importante reducción de la demanda de refrigeración. Esto provoca que las emisiones de refrigeración se reduzcan notablemente y por tanto hay una clara mejora en el resultado global, produciéndose la mejora en una letra: de G a F. Como nota a tener en cuenta, las emisiones de calefacción mejoran a pesar de haber un aumento de las demandas debido a la actualización en el procedimiento para la obtención de las prestaciones medias estacionales de equipos y sistemas de producción de frío y calor (13).

En este caso, con la introducción del clima alfa3 por parte del HE 2013, se observa en la Figura 3 como la demanda de calefacción desaparece y la de refrigeración se reduce notablemente. En cuanto a las emisiones, a pesar de que sucede lo mismos que con la demanda y que el valor global de emisiones de $\mathrm{CO} 2$ es mucho menor, la calificación final sigue siendo la peor, una $\mathrm{G}$, debido al alto valor de emisiones producido por el 60\% de las viviendas que cuentan con equipos de ACS eléctricos, algo que penaliza enormemente y que sin embargo su uso está enormemente extendido en las islas.

En este caso vuelve a percibirse en la Figura 4 como el valor de radiación solar no tiene ningún efecto sobre el resultado del certificado y como se nuevo los nuevos valores climáticos introducidos con el HE 2013 reducen enormemente los valores de demanda de refrigeración y emisiones, a pesar de que existe un leve repunte en la demanda de calefacción. El resultado global de emisiones de $\mathrm{CO} 2$ se reduce lo suficiente como para que se mejore una letra: de G a F.

Podemos ver en la Figura 5 un claro contraste en cuanto a la evolución que se ha producido en esta zona: mientras que con el CLIMCAN se produce una reducción en los valores de demanda y emisiones de calefacción y un aumento en los de refrigeración con respecto el HE 2016, con la llegada del HE 2013 nos encontramos ante la situación opuesta, es decir, un aumento en los valores de demanda y emisiones de calefacción y una muy notable reducción en los de refrigeración. En cuanto al valor de emisiones globales, es similar para los dos primeros mientras que con el HE 2013 se mejora una letra.

En este caso, cabe destacar que mientras el HE 2006 define esta zona como zona climática B3, CLIMCAN y HE 2013 lo definen como $\mathrm{C} 2$. Sin embargo los valores de demanda y emisiones expresados en la Figura 6 son más cercanos entre las simulaciones realizadas según los parámetros marcados por HE 2006 y CLIMCAN que por la del HE 2013. Esto se debe a que con esta última revisión del Documento Básico

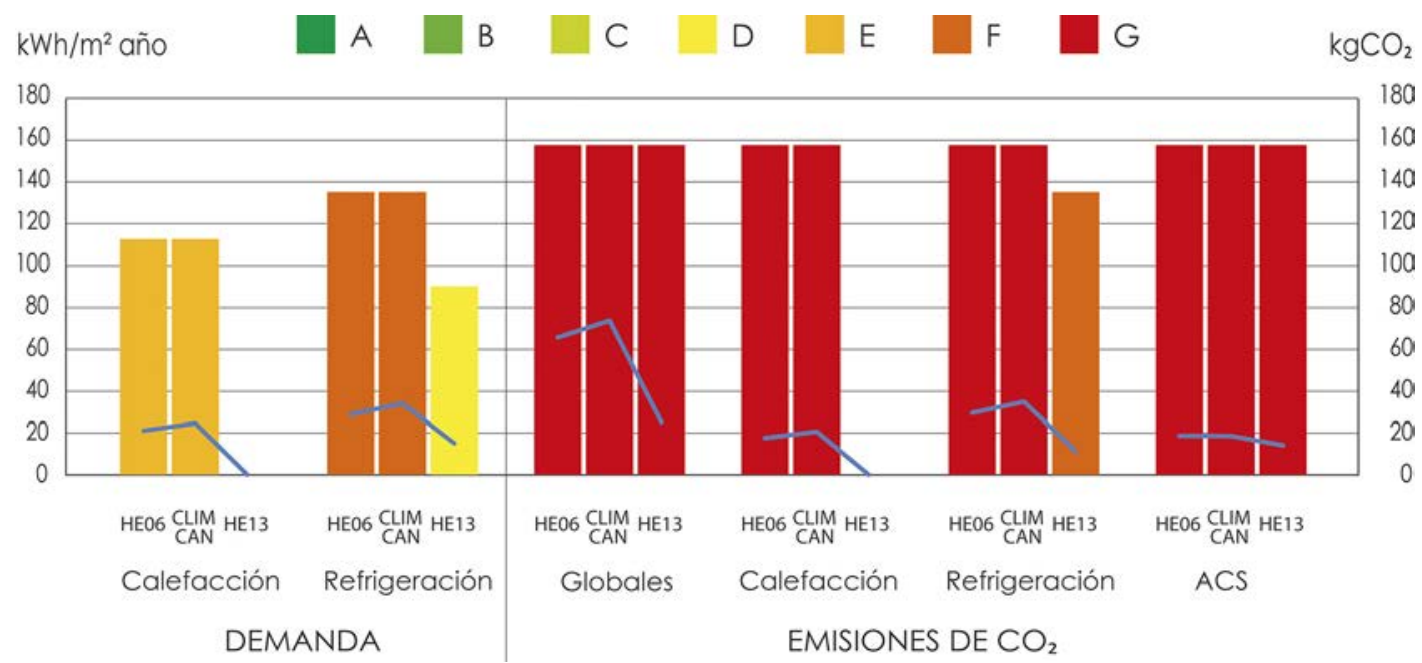

Figura 3. Comparativa de demandas y emisiones para un edificio situado en La Restinga.

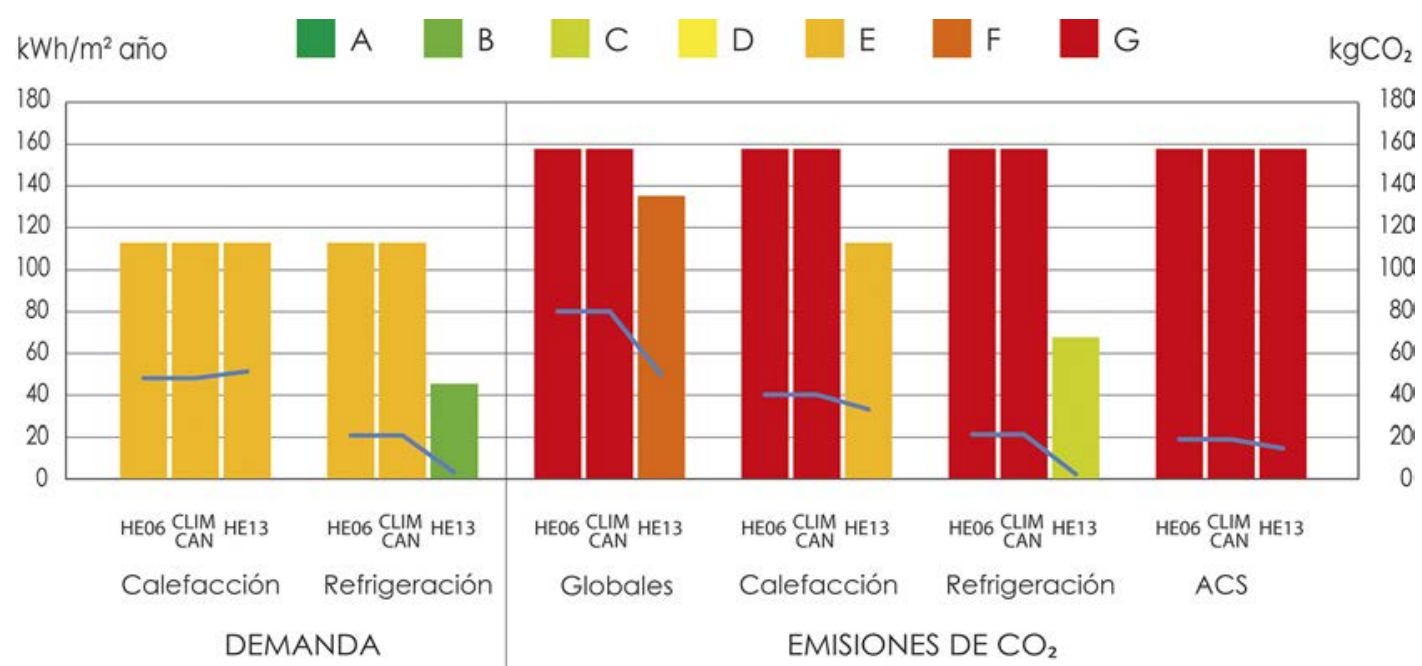

Figura 4. Comparativa de demandas y emisiones para un edificio situado en La Esperanza. 
del CTE, no sólo modifica las zonas climáticas, sino que se modifican los parámetros que caracterizan los climas de referencia, las correlaciones que se han establecido para determinar las severidades climáticas estacionales y los intervalos de severidades climáticas considerados para definir los climas de referencia.
La penúltima zona estudiada, tal como se muestra en la Figura 7, continúa en la misma línea que muchas de las anteriores, con valores similares entre el HE 2006 y el CLIMCAN y una importante reducción en los valores de demanda y emisiones de refrigeración para el HE 2013, que vuelve a conseguir la mejora de una letra en la calificación global de emisiones.

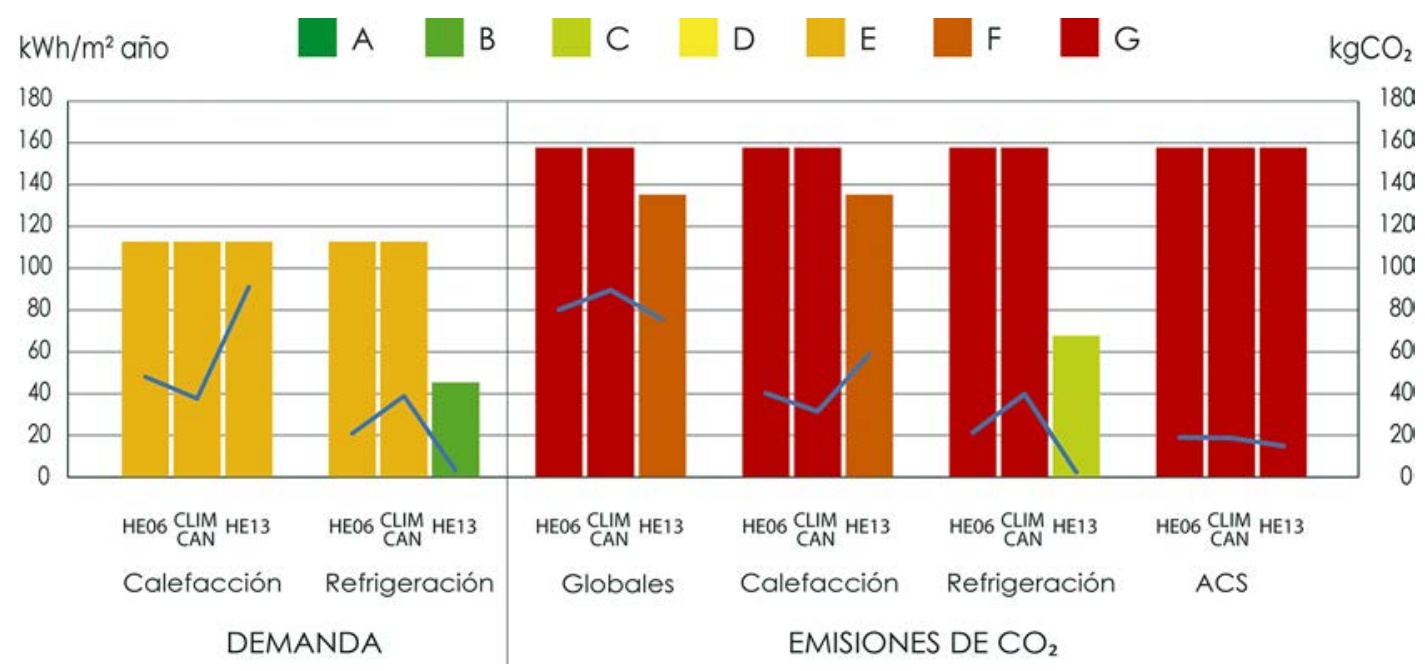

Figura 5. Comparativa de demandas y emisiones para un edificio situado en Vilaflor.

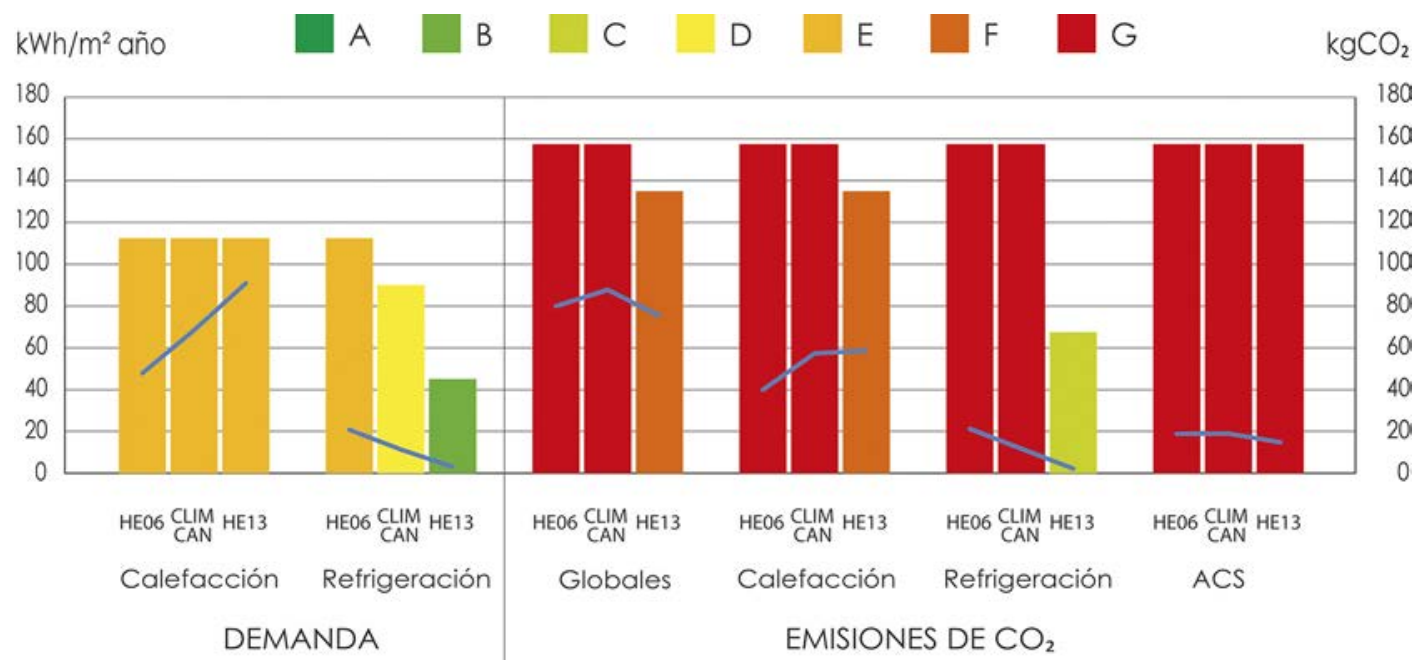

Figura 6. Comparativa de demandas y emisiones para un edificio situado en Malpaso.

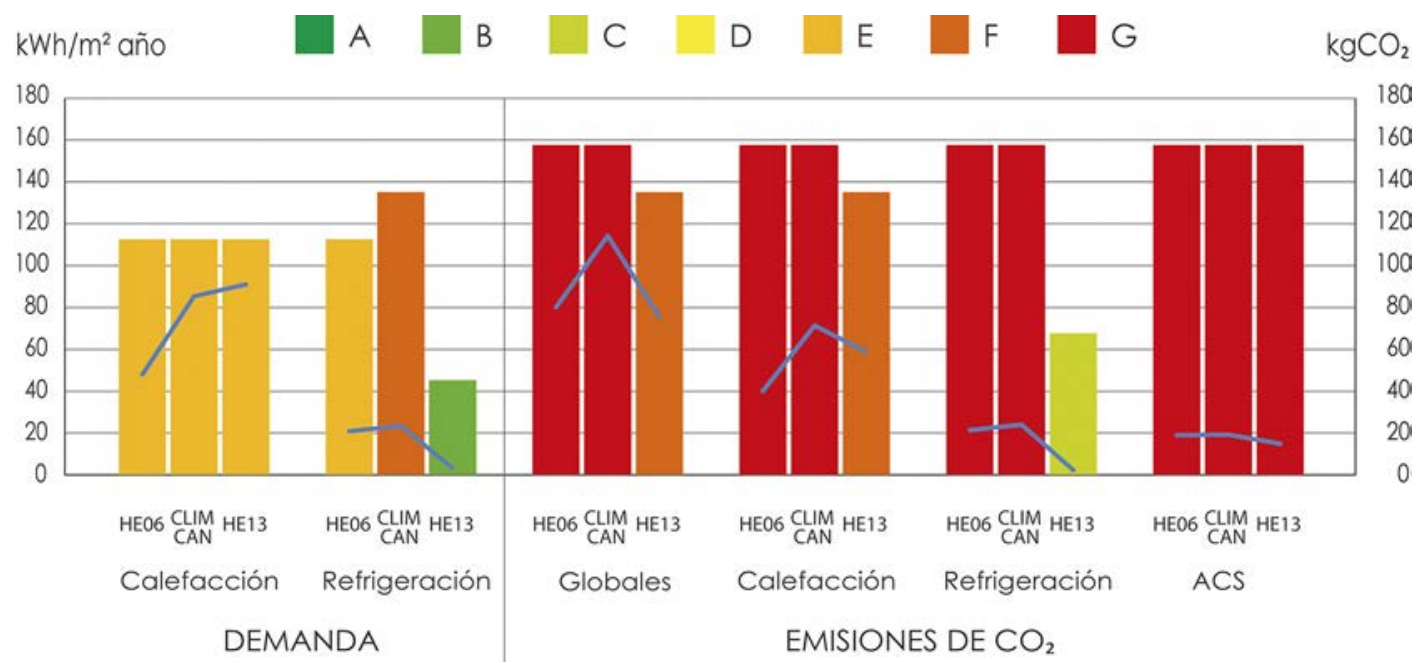

Figura 7. Comparativa de demandas y emisiones para un edificio situado en San José de Los Llanos. 


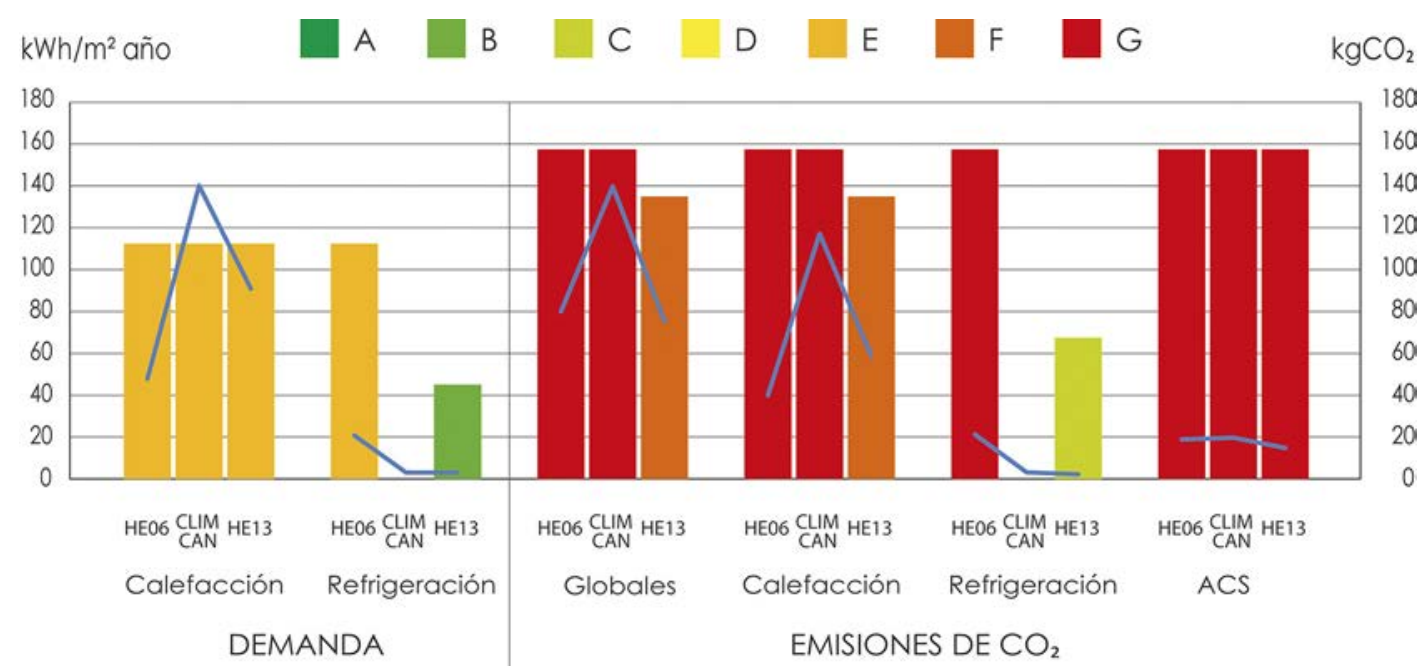

Figura 8. Comparativa de demandas y emisiones para un edificio situado en el Parador de Cañadas del Teide.

En esta última zona de estudio, puede observarse en la Figura 8, por primera vez como la zonificación climática marcada por el CLIMCAN tenemos valores de refrigeración (nulos) inferiores a los obtenidos según el HE 2013, que en todos los casos anteriores había obtenido los menores valores en este apartado. En contraposición, los valores de calefacción en la Zona climática marcada por el CLIMCAN son muy superiores a los obtenidos mediante ambos HE, lo que llevaría a recomendaciones de aislamiento térmico de mucho mayor espesor que los que propondrían los Documentos Básicos. De nuevo la certificación energética según el HE 2013 consigue la mejora de una letra en la calificación global de emisiones de $\mathrm{CO}_{2}$.

A partir de estas gráficas podemos analizar las consecuencias de aplicar las distintas zonificaciones climáticas a un mismo inmueble:

En cuanto a las demandas de calefacción, frente al escalón en dos niveles que presenta el HE 2006, el CLIMCAN presenta una evolución más lineal, pero existiendo demanda de calefacción en todos los casos. El HE 2013 corrige las existencia de demanda de calefacción en la zona costera de las islas aunque es menos detallado para el resto del territorio que el CLIMCAN.

En cuanto a las demandas de refrigeración, se mantienen similares entre el HE 2016 y el CLIMCAN, salvo que se anula en el caso de las cotas más altas del archipiélago y se produce una importante reducción en general con el HE 2013.

Las emisiones de $\mathrm{CO} 2$ de calefacción llevan la misma línea que las demandas, aunque en el caso del HE 2013, se mejora en una letra con respecto a los otros dos, a causa de la actualización de los coeficientes de paso y el procedimiento para la obtención de las prestaciones medias estacionales de equipos y sistemas de producción de frío y calor.

Las emisiones de refrigeración se reducen drásticamente en el HE 2013, debido lado a la modificación de las zonas climáticas, que modifican los parámetros que caracterizan los climas de referencia, las correlaciones que se han establecido para determinar las severidades climáticas estacionales y los intervalos de severidades climáticas considerados para definir los climas de referencia, que se suma al hecho de la actua- lización de los coeficientes de paso y el procedimiento para la obtención de las prestaciones medias estacionales de equipos y sistemas de producción de frío y calor.

Con todo esto tenemos que los nuevos valores de emisiones globales obtenidos con el HE 2013 son en general levemente inferiores que los que obteníamos con el HE 2006 y significativamente inferiores a los obtenidos aplicando el CLIMCAN.

\section{DISCUSIÓN DE RESULTADOS}

\subsection{Primera}

Cuando introducimos la zona climática, la herramienta informática para la certificación energética $\mathrm{CE}^{3} \mathrm{X}$, nos exige dos valores denominados: HE-1 y HE-4.

El primero de ellos es la severidad climática, es decir, la zona climática identificada mediante una letra, correspondiente a la severidad climática de invierno, (A, B, C, D, E), y un número, correspondiente a la severidad climática de verano $(1,2,3,4)$. La severidad climática combina los gradosdía y la radiación solar de la localidad.

Sin embargo, el segundo hace referencia a la Zona de Radiación solar global media diaria anual sobre superficie horizontal, es decir la energía procedente del sol o radiación solar directa e indirecta (global) que llega a una determinada superficie horizontal, tomando el valor anual como suma de valores medios diarios.

Si bien el Documento Reconocido CLIMCAN-010 pone énfasis en mejorar, ampliar y rectificar -en su caso- la información que describe el clima de las Islas Canarias para ambos valores (HE-1 y HE-3) cuando realizamos la certificación energética de un mismo inmueble siguiendo dos caracterizaciones de zonas climáticas en las que el valor de HE-1 se mantiene constante y sólo varía el de $\mathrm{HE}-4$, los resultados son exactamente iguales.

Por tanto descubrimos que el valor de la Zona de radiación solar (HE 4) no tiene ninguna influencia en el resultado de los certificados energéticos, algo que si influye mucho en las condiciones climáticas de los distintos microclimas de Canarias. 


\subsection{Segunda}

Si analizamos los valores de demandas de calefacción de las diferentes zonificaciones climáticas estudiadas podemos observar que mientras el HE 2006 es, como sabíamos, muy limitado en cuanto a variación climática, claramente alejado de la realidad, el HE 2013 desarrolla una función lineal para la costa y medianías, mostrándose finalmente plana a partir de los 100 metros. El CLINCAN es el que más variaciones climáticas o zonas climáticas desarrolla.

En cuanto a la demanda de refrigeración podemos observar que el HE 2006 mantiene el escalonamiento inverso al de calefacción, el CLIMCAN sigue siendo el que más variables desarrolla, aunque con valores cercanos al anterior pero reduciendo la demanda de refrigeración en las cotas más altas del archipiélago y el HE 2013 reduce a valores que tienden a cero la demanda de refrigeración en todo el archipiélago a excepción de unos valores algo superiores, pero todavía muy bajos en las zonas costeras.

Por tanto, tenemos que los valores de demanda tienden a ser más escalonados o estables en los HE mientras que el CLIMCAN desarrolla una estrategia de incremento más lineal y variado, lo que parece corresponderse más con las distintas circunstancias climáticas del archipiélago. La única zonificación climática que elimina la calefacción en las costas de las islas es el HE 2013.

\section{5•3. Tercera}

En relación al punto anterior podemos trasladar este hecho a la repercusión que dichas demandas tienen en las emisiones de $\mathrm{CO}_{2}$, dado que las emisiones por refrigeración van a ser también evidentemente nulas y las de calefacción en general menores que las del CLIMCAN y con unos valores similares a los del HE 2013 con la excepción de la zona alfa3 donde son nulas.

Por tanto podemos apreciar que la zonificación climática introducida por el HE 2013 mejora en prácticamente todos los casos estudiados el valor global de emisiones de $\mathrm{CO}_{2}$, reduciendo siempre los valores de refrigeración y tomando un leve aumento en los de calefacción, excepto en el nuevo clima alfa3, donde la demanda y por tanto los consumos de esta desaparece.

\subsection{Cuarta}

A pesar que la calidad constructiva en Canarias antes de la llegada del CTE era bastante mala, sin emplearse el aislamiento térmico en casi todo el territorio y con carpinterías metálicas de vidrio simple como sistema más usual, el benigno clima permitía unas condiciones climáticas dentro de gran parte de las viviendas, cercanas a las de confort, durante buena parte del año.

En este sentido, las demandas de calefacción y refrigeración en dichas viviendas son relativamente bajas y si estas se satisfacen con unos equipos con una eficiencia media, son suficientes para no emitir mucho $\mathrm{CO}_{2}$ a la atmósfera.

El problema se produce cuando atendemos a los equipos de ACS que en el archipiélago son prácticamente siempre equipos individuales siendo los más comunes los eléctricos -por efecto Joule- muy penalizados por el tipo de combustible que emplean y por su baja eficiencia energética.

Por tanto podemos aseverar que en el caso de las viviendas en Canarias, donde la demanda de calefacción y de refrigeración es generalmente baja, es el equipo de ACS el que condiciona de forma más contundente la calificación global de emisiones, siendo generalmente mala.

\subsection{Quinta}

Dado que la calificación energética es una herramienta que se emplea no sólo en las viviendas usadas, sino que también es un instrumento de proyecto en edificios nuevos, para poder hacerlos más sostenible, se puede dar el caso de que dos edificios proyectados bajo distintas zonificaciones climáticas (HE 2006, CLIMCAN o HE 2013), a pesar de haber obtenido como resultado con el mismo valor de emisiones globales y energéticas (imaginemos que ambos obtienen una calificación energética "A") van a ser más o menos sostenibles según estas zonificaciones se acerquen a la realidad; pudiendo incluso darse el caso de ser más contaminante un edificio "A" según el HE 2006 (y poco ajustado a la realidad climática donde se sitúa) que otro "B” según el CLIMCAN, por ejemplo).

Por tanto, un edificio en el que se haya puesto un gran esfuerzo de proyecto para obtener una alta eficiencia energética, puede no serlo tanto dado que se proyectó bajo una zona climática alejada de la realidad.

Del mismo modo, cuando se realiza un certificado a un edificio existente, éste no sólo califica la vivienda, sino que propone una serie de medidas de mejoras. Una incorrecta zonificación climática puede dar lugar a que se propongan o, peor aún, se materialicen unas obras de mejora, que no sólo no mejoren de forma cierta y efectiva la eficiencia energética del inmueble y reduzca sus emisiones de $\mathrm{CO}_{2}$, sino que se puede producir incluso un efecto contrario, dándose el caso que la inversión económica redunde en unos mayores consumos energéticos.

Por tanto podemos afirmar que la certificación energética pudiera premiar soluciones inadecuadas o innecesarias para el clima insular y en cambio no valorar otras que son más sostenibles, generando inmuebles contaminantes con alta calificación energética y por contra penalizando inmuebles bioclimáticos de baja o nulas emisiones de $\mathrm{CO}_{2}$ con una mala calificación energética, tanto en el caso de edificios nuevos como existentes.

\section{CONCLUSIONES}

Dado todo lo anterior, desde este estudio podemos exponer que el certificado energético, tal y como funciona actualmente, no tiene sentido en Canarias, lo que no quiere decir que este autor proponga su eliminación: todo lo contrario.

A la luz de los resultado obtenidos sería necesario darle una mayor profundidad a este documento informativo y que el mismo, de forma clara y precisa, es decir cualitativa y cuantitativamente, tal y como mide las demandas, consumos y emisiones asociadas de un inmueble, también evaluara el nivel de confort interior de las viviendas, de 
tal modo que un ciudadano no sólo no supiera cuales son los consumos energéticos y económicos previsibles del uso de una vivienda y las emisiones de $\mathrm{CO}_{2}$ al medio ambiente asociadas, sino que pudiera conocer el grado de confort higrotérmico que puede obtenerse en el interior de esa vivienda y cuáles son las medidas recomendadas para mejorarlo.

Para ello, también sería bueno que dicho certificado pudiera exponer cuales son las afecciones asociadas a las causas que provocan el disconfort estimado -es decir, que si por ejemplo, con la redacción del certificado se estima que la temperatura en verano va a estar por encima de la admisible, indique que esto puede suponer: deshidratación, calambres, arritmias,...- para que así el receptor del informe entendiera que los problemas de confort van más allá de una cuestión de comodidad, siendo causa muy importante para el correcto estado de nuestra salud, tomando la importancia que se merece.

Además este estudio, ampliado al resto del territorio nacional, podría ofrecer una información muy necesaria, ya que no sólo es importante conocer en qué regiones las viviendas consumen más o menos energía o son más contaminantes o menos, sino dónde las condiciones interiores de los edificios son más saludables o dañinas al organismo, para descubrir aquellos puntos negros de nuestra geografía nacional, donde los edificios son más insalubres.

Por tanto podemos concluir que la certificación energética debería sumar un nuevo dato en sus resultados, que en lugar de poner equipos por defectos para conseguir las condiciones de confort interior (o alternativamente a ello) expusiera las condiciones higrotérmicas a las que se llegaría en el interior del edificio con las características que presenta -de envolvente y equipos- para estimar situaciones de disconfort a lo largo del año y en consecuencia problemas o afecciones asociadas a las mismas que pudieran sufrir los usuarios y las medidas necesarias para poder mantener las condiciones de confort a lo largo de todo el año. Este sería un nuevo -y muy relevante- dato comparativo de cara a la elección de un inmueble en caso de compra o alquiler, así como una base de información importantísima de cara a la concesión de ayudas o al impuso de actuaciones de rehabilitación por parte de la Administración.

\section{AGRADECIMIENTOS}

A la arquitecta y Máster en Medio Ambiente y Arquitectura Bioclimática y en Planeamiento Urbano y Territorial, Teresa Eiroa Escalada, por sus consejos y sugerencias de cara a abordar y desarrollar este trabajo.

\section{REFERENCIAS}

(1) Ministerio de Vivienda (2006). Real Decreto 314/2006, de 17 de marzo, por el que se aprueba el Código Técnico de la Edificación. Boletín Oficial del Estado núm. 74, de 28 de marzo de 2006, pp. 11816 a 11831. Recuperado de http://www. boe.es/boe/dias/2006/03/28/pdfs/A11816-11831.pdf

(2) Canarias. Consejería de Empleo, Industria y Comercio (2008). Circular de la Dirección General de Energía $n^{\circ} 02 / 08$ de 10 de septiembre relativa a la zonificación climática aplicada por el programa de referencia para la obtención de la calificación de eficiencia energética de un edificio. Recuperado de http://www.gobcan.es/ceic/energia/doc/normativa/ eficienciaenergetica/circularzonasclim.pdf

(3) CTE-DR/oo8/11 (2011). Caracterización climática de las Islas Canarias para la aplicación del Código Técnico de la Edificación, CLIMCAN-010 y de su aplicación informática. Documento Reconocido del CTE, del Registro General del CTE, de acuerdo con lo dispuesto en la ORDEN VIV/1744/2008, de 9 de junio, por la que se regula el Registro General del Código Técnico de la Edificación. Inscrito el 11 de octubre 2011. Recuperado de https://www.codigotecnico.org/images/ stories/pdf/registroCTE/o1-documentos-reconocidos-CTE/CTE-DR-oo8-2011.zip

(4) Luxán García de Diego, M., Reymundo Izard, A. y Bango Yanes, M.C. (2006). CTE. Documento de Ahorro Energético. Recomendaciones para la aplicación del DB HE en los climas canarios. COACTFE: notas técnicas. Recuperado de https://docplayer.es/12440953-Cte-documento-de-ahorro-energetico-recomendaciones-para-la-aplicacion-del-db-heen-los-climas-canarios.html

(5) COAC (2010). Alegaciones al documento: Caracterización climática de las Islas Canarias para la aplicación del Código Técnico de la Edificación, CLIMCAN-010. Colegio Oficial Interinsular de Arquitectos de Canarias: Información Decano Alegaciones CLIMCAN. Recuperado de http://www.coacanarias.org/sites/default/files/Alegaciones.pdf

(6) Ministerio de Fomento (2013). Orden FOM/1635/2013, de 10 de septiembre, por la que se actualiza el Documento Básico DB-HE “Ahorro de Energía”, del Código Técnico de la Edificación, aprobado por Real Decreto 314/2006, de 17 de marzo. Boletín Oficial del Estado núm. 219, de 12 de septiembre de 2013, pp. 67137 a 67209. Recuperado de http://www. boe.es/boe/dias/2013/o9/12/pdfs/BOE-A-2013-9511.pdf

(7) Ministerio de Industria, Energía y Turismo (diciembre 2017). Estado de la certificación energética de los edificios (6º Informe). Recuperado de https://www.certificadosenergeticos.com/wp-content/uploads/2018/12/informe-seguimientocertificacion-energetica.pdf

(8) Ministerio de Industria, Energía y Turismo (2012) Procedimientos de certificación energética para edificios existentes. Informe ejecutivo. 2012. Recuperado de https://energia.gob.es/desarrollo/EficienciaEnergetica/CertificacionEnergetica/DocumentosReconocidos/Documents/Informe_ejecutivo_Herramientas_Certificacion_Edificios_Existentes.pdf

(9) Certicalia. ¿Son rentables las medidas de ahorro del certificado energético en Canarias? Certicalia: blog. Recuperado de https://www.certicalia.com/blog/son-rentables-las-medidas-de-ahorro-del-certificado-energetico-encanarias

(10) Martín del Toro, E. (2013). Canarias: El vergel de la mala construcción, Sustentable \& Sostenible. Recuperado de https:// blog.deltoroantunez.com/2013/12/canarias-vergel-de-mala-arquitectura.html 
(11) COAGC (2015). Modificación de documentos reconocidos para la certificación de la eficiencia energética de edificios y aceptación de certificados en registros de CCAA. 14 de diciembre de 2015. Ministerio de Industria, Energía y Turismo. Recuperado de http://arquitectosgrancanaria.es/medios/documents/edificacion/151217_nota.pdf

(12) Nota informativa sobre el procedimiento para la certificación de la eficiencia energética de edificios vigente desde el 14 de enero de 2016. Agosto de 2016. Ministerio de Industria, Energía y Turismo. Recuperado de https://energia.gob.es/desarrollo/EficienciaEnergetica/CertificacionEnergetica/DocumentosReconocidos/Documents/2016-08-04_NOTA\%20 INFORMATIVA.pdf

(13) IDAE (2015). Manual de fundamentos técnicos de calificación energética de edificios existentes CE3X. Redactado por EFINOVATIC y el Centro Nacional de Energías Renovables (CENER) para el Instituto para la Diversificación y Ahorro de la Energía (IDAE). Madrid: IDAE. Recuperado de http://www.coitivigo.es/historico-formacion/formacion_2012/ CIRC81/CE3X/Manual_fundamentos_tecnicos_CE3X_o5.pdf 